\title{
Inequalities for Higher Order Ising Spins and for Continuum Fluids *
}

\author{
Joel L. Lebowitz \\ Belfer Graduate School of Science, Yeshiva University, New York, N.Y., USA \\ James L. Monroe ${ }^{\star \star}$ \\ Dept. of Physics, Columbia University, New York, N.Y., USA
}

Received July 1, 1972

\begin{abstract}
The recently derived Fortuin, Kasteleyn and Ginibre (FKG) inequalities for lattice gasses are investigated for higher order Ising spin systems and multi-component lattice gasses. Conditions are given for the validity of the FKG inequalities for higher order spin systems with Hamiltonians of the form used recently as models for various physical systems, e.g. $\mathrm{He}^{3}-\mathrm{He}^{4}$ mixtures. We also investigate various inequalities for binary lattice gases and show how these can be carried over to continuum systems.
\end{abstract}

\section{Introduction}

In recent years a number of physical phenomena have been studied where systems consisting of Ising type particles of spin one, $S_{i}=2,0,-2$, or higher spin have been used as models. Some examples of such phenomena are a) the phase transitions of $\mathrm{UO}_{2}$ [1] and $\left.\mathrm{DyV0}_{4}[2], \mathrm{b}\right)$ annealed alloys of magnetic and non-magnetic atoms [3], c) the separation of components in a classical mixture $[4,5]$, and d) the $\lambda$-transition and phase separation in $\mathrm{He}^{3}-\mathrm{He}^{4}$ mixtures [6]. Higher order spin systems have also been investigated in order to gain insight into the general nature and existence of phase transitions [7].

An interesting question in these investigations is the extent to which the many results known for simple spin $\frac{1}{2}$ Ising systems, $S_{i}= \pm 1$, with ferromagnetic interactions remain valid for these higher spin systems. This question was partly answered by Griffiths [7] who showed that every higher order Ising spin system can be "mapped" into some spin $\frac{1}{2}$ Ising systems. Higher spin systems with purely ferromagnetic interactions were reduced to spin $\frac{1}{2}$ systems with the same property. This showed immediately that the Griffiths [8], Kelly and Sherman [9] (GKS) inequalities for spin $\frac{1}{2}$ Ising systems with ferromagnetic interactions re-

* Supported in part by U.S.A.F.O.S.R. \# F44620-71-C-0013.

$\star \star$ N.S.F. Graduate Trainee. 
mained valid also for higher spins. Unfortunately though most of the higher spin systems used as physical models do not have all their interactions ferromagnetic. The GKS inequalities do not therefore apply to these systems. Recently however Fortuin, Kasteleyn, and Ginibre [10] (FKG) derived some very interesting new inequalities for certain lattice gases. The FKG inequalities apply in many cases in which the GKS do not (and vice versa). In this note we find conditions for the validity of the FKG inequalities for higher spin systems with Hamiltonians of the type considered in the physical models.

We find for example that the FKG inequalities can hold for multicomponent lattice gases even in the presence of extended hard core potentials between particles of different species on the lattice. This permits an extension of these inequalities to continuum systems which may be considered as limiting cases of lattice systems. An example of such a system is the Lebowitz-Gallavotti [11] lattice model which becomes the Widom-Rowlinson [12] continuum model. (The existence of phase transitions in such continuum models was recently proven by Ruelle [13], and Lebowitz and Lieb [14].) We also show that some of the inequalities remain valid even for systems with extended hard core interactions between particles of the same species. These too are extended to continuum systems.

We begin in Section 2 by introducing a general lattice spin system Hamiltonian and also some general notation. In Section 3 we derive sufficient conditions on the interaction coefficients of this Hamiltonian for the FKG inequalities to hold. We then show how the spin one system becomes, through a particular choice of interaction coefficients isomorphic to a binary lattice gas having extended hard core exclusion between $A$ and $B$ particles; $S_{i}=2\left(S_{i}=-2\right)$ denotes an $A$-type ( $B$-type) particle. This system is designated as the $A-B$ system and satisfies the FKG inequalities. In Section 4 we consider binary systems which have extended hard core exclusion between like particles as well as unlike particles. For these systems neither the GKS nor FKG inequalities are valid. We show however the validity of a more restricted set of inequalities. In the concluding section, Section 5, the inequalities of the previous sections for binary lattice systems are extended to the corresponding continuum systems.

\section{Description of the General System}

The general system which we consider in the following sections consists of an arbitrary lattice in $v$ dimensions, enclosed in a region $V$ and containing $|V|$ lattice sites. On each site there is an Ising spin variable 
$S_{i}$, where $S_{i}$ can take on the values $p, p-2, \ldots,-p+2,-p$, where $p$ is a positive integer. The Hamiltonian of this spin system which we shall consider has the form

$$
H(\{S\})=-\sum_{i<j} J(i, j) S_{i} S_{j}-\sum_{i<j} \gamma(i, j) S_{i}^{2} S_{j}^{2}-\sum_{i} h(i) S_{i}-\sum_{i} \mu(i) S_{i}^{2} \quad i, j \in V
$$

where $h(i)$ may be thought of as an external magnetic field. Setting $S_{i}=2 n_{i}-p, n_{i}=0,1, \ldots, p$ this system is equivalent to a lattice gas system where each site may be occupied by 0 to $p$ particles.

For the special case where $p=3$ and $J(i, j), \gamma(i, j)$ different from zero only for nearest neighbor pairs we have the system used as a model for $D y \mathrm{VO}_{4}$. If we consider the case where $p=2$ we can with proper choices of the four coefficients, $J(i, j), \gamma(i, j), h(i)$ and $\mu(i)$, in Eq. (2.1) obtain the other lattice systems mentioned in the introduction.

Throughout the paper we define $\Gamma$ as the set of all $(2 p+1)^{|V|}$ states of particular system under consideration and we denote specified states by $x, y \in \Gamma$. The thermal average, indicated by brackets, of any function $f$ defined on $\Gamma$ is

where

$$
\langle f\rangle=Z_{V}^{-1} \sum_{x \in \Gamma} f(x) e^{-\beta H(x)}
$$

$$
Z_{V}=\sum_{x \in \Gamma} e^{-\beta H(x)}
$$

\section{GKS and FKG Inequalities}

As mentioned in Section 1 it follows from the general results of Griffiths [7] that the GKS inequalities

$$
\left\langle S_{A} S_{B}\right\rangle-\left\langle S_{A}\right\rangle\left\langle S_{B}\right\rangle \geqq 0
$$

where $S_{A}=\prod_{i \in A} S_{i}, S_{B}=\prod_{i \in B} S_{i}, A, B \subset V$, hold for Ising systems with arbitrary spins whenever all the coefficients of the spin variables in (2.1) are non-negative. (By symmetry they also hold when $h(i) \leqq 0$ for all $i \in V$ and the other coefficients are non-negative.)

We turn now to the conditions on the coefficients of (2.1) necessary for the FKG inequalities to hold. We shall need the following notation: For any $x, y \in \Gamma$ let $S_{i}(x), S_{i}(y)$ be the spin on the $i$-th site, $i \in V$, for the $x$ and $y$ states respectively. We define $x \geqq y$ if for every $i \in V S_{i}(x) \geqq S_{i}(y)$. This definition establishes $\Gamma$ as a partially ordered set. Next we define $f(x)$ to be an increasing (decreasing) function on $\Gamma$ if $f(x) \geqq f(y)$ $(f(x) \leqq f(y))$ for any two states $x$ and $y$ such that $x \geqq y$. The FKG ine- 
qualities and the conditions on (2.1) for which they are valid may now be stated as follows:

Theorem 1. Let $f$ and $g$ be both increasing (decreasing) functions on $\Gamma$. Then

whenever

$$
\langle f g\rangle-\langle f\rangle\langle g\rangle \geqq 0
$$

$$
J(i, j) \geqq(2 p-2)^{2}|\gamma(i, j)| \text { for all } i, j \in V .
$$

Proof. The result follows from Proposition 1 of Ref. [10] after showing first that the states of the system form a distributive lattice and second that the stated conditions on the $J(i, j)$ 's and $\gamma(i, j)$ 's are sufficient to insure the validity of the inequality

$$
\mu(x \vee y) \mu(x \wedge y) \geqq \mu(x) \mu(y)
$$

where $\mu(x)=\exp [-\beta H(x)]$ and $x \vee y(x \wedge y)$ signifies the state which is the least upper bound (greatest lower bound) of the states $x$ and $y$. The least upper bound, $x \vee y$, is formed by taking the maximum spin between $S_{i}(x)$ and $S_{i}(y)$ for all $i \in V$ and $x \wedge y$ is formed by taking the minimum spin i.e., $S_{i}(x \vee y)=\max \left[S_{i}(x), S_{i}(y)\right]$ and $S_{i}(x \wedge y)=\min \left[S_{i}(x), S_{i}(y)\right]$.

Fortuin et al. [10] noted in their paper that the states of a lattice gas having as many as $p$ particles on a site or the corresponding states of the spin- $p / 2$ system form a distributive lattice. The proof is simple. By the definition we see immediately that the states form a lattice. To prove the distributive property of $\Gamma$ we must show that for any $x, y, z \in \Gamma$ the operations satisfy either of the two equivalent conditions

$$
\begin{aligned}
& x \wedge(y \vee z)=(x \wedge y) \vee(x \wedge z), \\
& x \vee(y \wedge z)=(x \vee y) \wedge(x \vee z) .
\end{aligned}
$$

In terms of the spin system we must have, choosing to verify the first of the two conditions, that for any site $i \in V$

$$
\begin{aligned}
\min & \left\{S_{i}(x), \max \left[S_{i}(y), S_{i}(z)\right]\right\} \\
& =\max \left\{\min \left[S_{i}(x), S_{i}(y)\right], \min \left[S_{i}(x), S_{i}(z)\right]\right\} .
\end{aligned}
$$

The $S_{i}(x), S_{i}(y)$, and $S_{i}(z)$ have 13 possible orderings $\left(1\right.$ where $S_{i}(x)$ $=S_{i}(y)=S_{i}(z), 6$ where two of the spin values are equal, and 6 where all three spin values differ) and it is then a somewhat tedious but trivial matter to verify the correctness of Eq. (3.4). Since the distributive property holds for each site $i \in V$ it holds for all $V$ and therefore, the set of all states, $\Gamma$, forms a distributive lattice.

We now show that the condition $J(i, j) \geqq(2 p-2)^{2}|\gamma(i, j)|$ is the maximum restrictive condition imposed by the inequality (3.3). First we 
note that when $x \geqq y$, i.e. two ordered states, then (3.3) holds as an equality since $\mu(x \vee y)=\mu(x)$ and $\mu(x \wedge y)=\mu(y)$. Next we must consider two unordered states $x$ and $y$. The one-body interactions in (2.1), $h(i) S_{i}$ and $\mu(i) S_{i}^{2}$, have no effect on the inequality (3.3). This is because each $S_{i}(x), S_{i}(y), S_{i}^{2}(x)$ and $S_{i}^{2}(y)$ appearing on the right hand side of the inequality also appears on the left hand side and hence cancel each other. We are then left with the pair interactions $J(i, j) S_{i} S_{j}$ and $\gamma(i, j) S_{i}^{2} S_{j}^{2}$ and (3.3) becomes

$$
\begin{gathered}
A B C D \geqq E(x) E(y), \\
A=\exp \beta \sum_{i<j} J(i, j)\left(\min \left[S_{i}(x), S_{i}(y)\right]\right)\left(\min \left[S_{j}(x), S_{j}(y)\right]\right), \\
B=\exp \beta \sum_{i<j} \gamma(i, j)\left(\min \left[S_{i}(x), S_{i}(y)\right]\right)^{2}\left(\min \left[S_{j}(x), S_{j}(y)\right]\right)^{2}, \\
E(z)=\exp \beta\left\{\sum_{i<j} J(i, j) S_{i}(z) S_{i}(z)+\sum_{i<j} \gamma(i, j) S_{i}^{2}(z) S_{j}^{2}(z)\right\}, \quad z=x \text { or } y .
\end{gathered}
$$

$C$ and $D$ are obtained from $A$ and $B$ respectively by substituting max for $\mathrm{min}$ in their definitions.

The inequality (3.7) will clearly be established if it holds for each term in the sum over pairs in the exponents. Looking now at a particular pair of sites $i, j$ the inequality in (3.7) becomes an equality if $S_{i}(x) \geqq S_{i}(y)$ and $S_{j}(x) \geqq S_{j}(y)$ or $S_{i}(x) \leqq S_{i}(y)$ and $S_{j}(x) \leqq S_{j}(y)$. Therefore we need only consider two sites $i, j \in V$ where these sites are unordered for the states $x$ and $y$. Let $S_{i}(x)=a, S_{j}(x)=b, S_{i}(y)=c$, and $S_{j}(y)=d$. Since the pairs of sites are unordered for the states $x$ and $y$ we must have $a<c$ and $b>d$ or $a>c$ and $b<d$. In either case the required inequality is,

This gives

$$
\begin{aligned}
& e^{\beta J(i j) a d} e^{\beta J(i j) b c} e^{\beta \gamma(i j) a^{2} d^{2}} e^{\beta \gamma(i j) b^{2} c^{2}} \\
& \quad \geqq e^{\beta J(i j) a b} e^{\beta J(i j) c d} e^{\beta \gamma(i j) a^{2} b^{2}} e^{\beta \gamma(i j) c^{2} d^{2}} .
\end{aligned}
$$

$$
J(i, j) \geqq-(a+c)(b+d) \gamma(i, j) .
$$

We must now vary $a, b, c$, and $d$ subject to the conditions $a>c$ and $b<d$ or vice versa and thereby obtain conditions on the coefficients $J(i, j)$ and $\gamma(i, j)$. The most restrictive condition is obtained when the value of the right hand side of Eq. (3.9) is at its maximum value. For $\gamma(i, j)$ negative the maximum value of the right hand side of (3.9) occurs when $a=p$, $b=p-2, c=p-2$ and $d=p$. For $\gamma(i, j)$ positive the desired condition is $a=p, b=-p, c=p-2$, and $d=-p+2$. Hence (3.3) will hold whenever $J(i, j) \geqq(2 p-2)^{2}|\gamma(i, j)|$, and we have proven Theorem 1 .

We note that the FGK inequalities are satisfied for values of the interaction coefficients much different from those necessary for the GKS inequalities. There are no conditions on the one-body terms $\mu(i)$ and $h(i)$ and we are able to consider cases where $\gamma(i, j)$ is negative. 
At this point we wish to consider explicitely a particular set of values of the interaction coefficients for a spin one $\left(S_{i}=2,0,-2\right)$ system. Let us interpret $S_{i}=+2(-2)$ to represent the presence of an $A$-type ( $B$-type) particle at the $i$-th site while $S_{i}=0$ corresponds to the $i$-th site being unoccupied. For the spin-one case the conditions of Theorem 1 require that $J(i, j) \geqq 4|\gamma(i, j)|$. We now set $J(i, j)=-4 \gamma(i, j) \geqq 0$ for all $|r(i, j)| \leqq d$ where $r(i, j)$ is the vector between the $i$-th and $j$-th sites. For sites $i$ and $j$ with $|r(i, j)|>d$ we make no restrictions on the values for $J(i, j)$ and $\gamma(i . j)$ other than that the inequality $J(i, j) \geqq 4|\gamma(i, j)|$ is satisfied. The Hamiltonian (2.1) now assumes the form

$$
\begin{aligned}
H= & -\Sigma^{\prime} J(i, j) S_{i} S_{j}\left(1-\frac{1}{4} S_{i} S_{j}\right)-\Sigma^{\prime \prime} J(i, j) S_{i} S_{j} \\
& -\Sigma^{\prime \prime} \gamma(i, j) S_{i}^{2} S_{j}^{2}-\Sigma h(i) S_{i}-\Sigma \mu(i) S_{i}^{2}
\end{aligned}
$$

where $\Sigma^{\prime}$ and $\Sigma^{\prime \prime}$ indicate summations over all pairs of sites for which $|r(i, j)| \leqq d$ and $|r(i, j)|>d$ respectively. Letting now $J(i, j) \rightarrow \infty$ for $|r(i, j)| \leqq d$ we obtain a system in which $A$ and $B$ particles exclude unlike particles from a sphere about their centers of radius $d$. We shall denote this as the $A-B$ system. If $J(i, j)$ and $\gamma(i, j)$ are zero for $|r(i, j)|>d$ then we have the lattice gas system for which Gallovatti and Lebowitz have proven the existence of a phase transition. This system has as it's continuum analog the Widom-Rowlinson model. We shall prove the existence of the above correlation inequalities for this continuum model in Section 5 .

\section{Restricted Inequalities}

We shall now consider a spin-one or binary lattice gas system in which there are extended hard cores of radius $d$ not only between $A-B$ pairs but also between $A-A$ and $B-B$ pairs. We shall label such a system the $A^{\prime}-B^{\prime}$ system. This system may be obtained by letting $\gamma(i, j) \rightarrow-\infty$ in the Hamiltonian (2.1) for $|r(i, j)| \leqq d$. For the $A^{\prime}-B^{\prime}$ system neither the GKS or FKG inequalities are valid. Nevertheless we will prove the existence of a restricted set of inequalities for this system whenever the interactions for $|r(i, j)|>d$ satisfy the conditions for the applicability of the GKS or FKG inequalities to spin- $\frac{1}{2}$ systems.

For any "permissable" configuration, $x$, let $R(x) \subset V$ be the set of all sites occupied by an $A\left(S_{i}=+2\right)$ or $B\left(S_{i}=-2\right)$ particle. By "permissable" we mean configurations where no particles are within a distance $d$ of one another. Note the transformation $x \rightarrow R(x)$ is many to one. For any function $f$ of the spin variable or of the occupation numbers in lattice 
gas language the thermal average can be written as

$$
\langle f\rangle=Z^{-1} \sum_{R \subset V} \sum_{\substack{S_{\alpha}= \pm 2 \\ \alpha=1,2, \cdots,|R|}} f e^{-\beta H},
$$

where $|R|$ is the number of sites in $R$. This may be rewritten as

$$
\langle f\rangle=\sum_{R \subset V} P(R)\left\{Z(R)^{-1} \sum_{\substack{S_{\alpha}= \pm 2 \\ \alpha=1,2, \ldots,|R|}} f e^{-\beta H}\right\}=\sum_{R \subset V} P(R)\langle f ; R\rangle .
$$

Here $\langle f ; R\rangle$ is a conditional thermal average of $f$ when we are given the set $R$ of occupied sites, $P(R)$ is the probability of having the $R$ sites occupied,

$$
P(R)=Z^{-1} \sum_{x}^{\prime} e^{-\beta H(x)}=Z(R) / Z .
$$

The prime indicates a summation only over those states $x$ which have the set $R$ as the occupied sites.

It is seen from (4.2) that $\langle f: R\rangle$ is the average of $f$ with respect to a spin $\frac{1}{2}$ Ising system, $S_{i}= \pm 2, i \in R$. Hence if the interaction $J(i, j) \geqq 0$ for all $i, j \in R$ then the $F K G$ inequalities are valid for this subsystem and if in addition $h(i)>0$ (or $h(i)<0$ ) for all $i \in R$ then the GKS inequalities too are valid. (Note that there is no constraint on the form of $\gamma(i, j)$ or $\mu(i)$ in (2.1).) These inequalities are of the form

$$
\langle f g ; R\rangle-\langle f ; R\rangle\langle g ; R\rangle \geqq 0 .
$$

where $f$ and $g$ have the form appropriate for the different inequalities. We shall use (4.4) to derive linear inequalities of the form

$$
\langle F ; R\rangle \geqq\langle G ; R\rangle
$$

where $F$ and $G$ are new functions of the states $x$. When (4.5) holds for all $R \subset V,(P(R) \neq 0)$ we can multiply both sides of (4.5) by $P(R)$ and sum over all $R$ to obtain the desired result

$$
\langle F\rangle \geqq\langle G\rangle \text {. }
$$

We now give an example of such an inequality starting with the FKG inequalities and therefore only require that $J(i, j) \geqq 0$ for all $i, j \in V$, $|r(i, j)|>d$. We define $\left\langle\varrho_{A}(i)\right\rangle\left(\left\langle\varrho_{B}(i)\right\rangle\right)$ as the density of $A(B)$ particles on the $i$-th site. In terms of the spin variables, $S_{i}$, we have

$$
\varrho_{A}(i)=\frac{S_{i}\left(S_{i}+2\right)}{8} \text { and } \varrho_{B}(i)=\frac{S_{i}\left(S_{i}-2\right)}{8} .
$$


Since $\varrho_{A}(i)$ and $-\varrho_{B}(i)$ are increasing functions of $x$ we have for any $R \subset V$,

and

$$
\left\langle\varrho_{A}(i) \varrho_{B}(j) ; R\right\rangle \leqq\left\langle\varrho_{A}(i) ; R\right\rangle\left\langle\varrho_{B}(j) ; R\right\rangle
$$

$$
\left\langle\varrho_{B}(i) \varrho_{A}(j) ; R\right\rangle \leqq\left\langle\varrho_{B}(i) ; R\right\rangle\left\langle\varrho_{A}(j ; R\rangle .\right.
$$

Multiplying the two inequalities together and then using the FKG inequality

$$
\left\langle\varrho_{A}(i) \varrho_{A}(j) ; R\right\rangle \geqq\left\langle\varrho_{A}(i) ; R\right\rangle\left\langle\varrho_{A}(j) ; R\right\rangle
$$

and the similar inequality for $\varrho_{B}(i)$ and $\varrho_{B}(j)$ we have

$$
\left\langle\varrho_{A}(i) \varrho_{B}(j) ; R\right\rangle\left\langle\varrho_{B}(i) \varrho_{A}(j) ; R\right\rangle \leqq\left\langle\varrho_{A}(i) \varrho_{A}(j) ; R\right\rangle\left\langle\varrho_{B}(i) \varrho_{B}(j) ; R\right\rangle \text {. }
$$

Until now we have placed no restriction on the one-body interactions $\mu(i)$ and $h(i)$. We now restrict ourselves to the case of a uniform periodic system, i.e. $h(i)=h, \mu(i)=\mu, i \in V$, and the potentials $J(i, j)$ and $\gamma(i, j)$ translationally invariant and satisfy periodic boundary conditions (when $h(i)=0$ these requirements of uniformity are not necessary). With these restrictions we have then that the two thermal averages on the left side of inequality (4.10) are equal.

Hence we have

$$
\begin{aligned}
\left\langle\varrho_{A}(i) \varrho_{B}(j) ; R\right\rangle & \leqq\left\{\left\langle\varrho_{A}(i) \varrho_{A}(j) ; R\right\rangle\left\langle\varrho_{B}(i) \varrho_{B}(j) ; R\right\rangle\right\}^{\frac{1}{2}} \\
& \leqq \frac{1}{2}\left\{\left\langle\varrho_{A}(i) \varrho_{A}(j) ; R\right\rangle+\left\langle\varrho_{B}(i) \varrho_{B}(j) ; R\right\rangle\right\} .
\end{aligned}
$$

Multiplying (4.11) by $P(R)$ and then summing over all possible $R$ we obtain

$$
\left\langle\varrho_{A}(i) \varrho_{B}(j)\right\rangle \leqq \frac{1}{2}\left[\left\langle\varrho_{A}(i) \varrho_{A}(j)\right\rangle+\left\langle\varrho_{B}(i) \varrho_{B}(j)\right\rangle\right] .
$$

Clearly other inequalities may be derived using these procedures the main requirement always being to first obtain an inequality for the subsystem $R$ in the form of Eq. (4.5).

We note that for systems where in addition to the $A^{\prime}-B^{\prime}$ system's hard core exclusion of radius $d$ we also have an additional exclusion between unlike, $A-B$, pairs to a greater radius which we call $D$ then the inequalities of (4.6) still remain valid if we begin with the FKG inequalities in (4.4). The inequalities hold for such systems because the additional hard core interaction is a result of requiring $J(i, j)=4 \gamma(i, j)$ and then letting $J(i, j) \rightarrow+\infty$ for $d<|r(i, j)| \leqq D$ and this is clearly allowable for the FKG inequalities.

\section{Inequalities for Continuum Systems}

In this section we make use of the results for the lattice systems of Sections 3 and 4 to derive similar inequalities for continuum systems. The transition from lattice systems to continuum systems is accom- 
plished by considering a sequence of cubical lattices with spacing $\delta$ in a given domain $V \subset \mathbb{R}^{v}$ and then letting the lattice spacing, $\delta$, go to zero while keeping the interaction potentials fixed as a function of $r_{i j}$ the Euclidian distance between the $i$-th and $j$-th sites.

We consider a continuum system contained in an open bounded set (domain) $V \subset \mathbb{R}^{v}$. The system contains two molecular species (the case where more than two species exists can be treated in a similar fashion) labeled by $\alpha=-2,2$ where $\alpha=2$ will denote on $A$ particle and $\alpha=-2$ a $B$ particle. Each of the molecular species has a fugacity, $z_{\alpha}$, which may be a function of position and is given by

$$
z_{\alpha}(\boldsymbol{r})=\exp \beta\left[\mu(\boldsymbol{r}) \alpha^{2}+h(\boldsymbol{r}) \alpha\right], \quad \alpha=-2,2, \boldsymbol{r} \in V .
$$

There is also an interaction potential $\phi_{\alpha \alpha^{\prime}}\left(\left|\boldsymbol{r}-\boldsymbol{r}^{\prime}\right|\right)$ between a molecule of species $\alpha$ at position $r$ and a molecule of species $\alpha^{\prime}$ at $\boldsymbol{r}^{\prime}$ which is given by

$$
\phi_{\alpha \alpha^{\prime}}\left(\left|\boldsymbol{r}-\boldsymbol{r}^{\prime}\right|\right)=-J\left(\left|\boldsymbol{r}-\boldsymbol{r}^{\prime}\right|\right) \alpha \alpha^{\prime}-\gamma\left(\left|\boldsymbol{r}-\boldsymbol{r}^{\prime}\right|\right) \alpha^{2} \alpha^{\prime 2} .
$$

We assume at this point that the functions $\exp -\beta \phi_{\alpha \alpha^{\prime}}\left(\left|\boldsymbol{r}-\boldsymbol{r}^{\prime}\right|\right)$ and $z_{\alpha}(\boldsymbol{r})$ are piece-wise continuous functions and satisfy the usual stability condition necessary to insure the existence of thermodynamic behavior, i.e., the total potential energy for an arbitrary configuration of $N$ particles has a lower bound of the form $-B N$, where $B<\infty$ and is independent of $N$. It is apparent with these definitions of the one and two-body interactions that this system is the continuum analog of the spin-one lattice system with the Hamiltonian of Eq. (2.1).

To make more explicit the correspondence between the lattice gas and continuum system let $X$ specify a configuration of the continuum system and let $\omega$ be an open set in $\mathbb{R}^{v}, \omega \subset V$. Define now the function $s_{c}(X ; \alpha, \omega)$ to equal the number of particles of species $\alpha$ which are in $\omega$ when the configuration of the system is specified by $X$ (cf. Ruelle [15]). In a similar way we define for the lattice gas the function

$$
s_{\delta}(x ; \alpha, \omega)=\sum_{i \in \omega} \varrho_{\alpha}(i)
$$

where $\varrho_{\alpha}(i)=1$ if the spin variable on the $i$-th site of $\omega$ is equal to $\alpha$ and is zero otherwise $\left(\varrho_{2}(i) \equiv \varrho_{A}(i), \varrho_{-2}(i) \equiv \varrho_{B}(i)\right.$ given by Eq. (4.7)); the sum is over all lattice sites $i$ which lie in the domain $\omega$ hence $s_{\delta}(x ; \delta, \omega)$ depends on the spacing $\delta$ of the lattice. It is now easily verified that under the conditions specified earlier the expectation value, in the continuum system grand ensemble, of any continuous function of the $s_{c}(X ; \alpha, \omega)$ 's, $f\left(\left\{s_{c}(X ; \alpha, \omega\}\right)\right.$, is equal to the limit, as $\delta \rightarrow 0$, of the expectation value of $f\left(\left\{s_{\delta}(x ; \alpha, \omega)\right\}\right)$. The Hamiltonian $H(x)$ of $(2.1)$, with $S_{i}=2,0,-2$, to be used in (2.2) and (2.3) for obtaining the expectation value of $f\left(\left\{s_{\delta}(x ; \alpha, \omega)\right\}\right)$ 
is related to the continuum functions defined in (5.1) and (5.2) in the following way

$$
\begin{gathered}
J(i, j)=J\left(\left|\boldsymbol{r}_{i}-\boldsymbol{r}_{j}\right|\right), \quad \gamma(i, j)=\gamma\left(\left|\boldsymbol{r}_{i}-\boldsymbol{r}_{j}\right|\right), \quad h(i)=h\left(\boldsymbol{r}_{i}\right), \\
\mu(i)=\mu\left(\boldsymbol{r}_{i}\right)+\beta^{-1} \ln \delta^{v}
\end{gathered}
$$

where $\boldsymbol{r}_{i}$ and $\boldsymbol{r}_{j}$ are the position vectors of the $i$-th and $j$-th lattice sites respectively and with the introduction of the logarithm in (5.4), (2.2), and (2.3) become Riemann sums of $f(x) e^{-\beta H(x)}$ and $e^{-\beta H(x)}$ respectively. These Riemann sums go over into the appropriate continuum integrals when $\delta \rightarrow 0$.

It is seen now that whenever $J(r) \geqq 4|\gamma(r)|$ then the FKG lattice inequalities hold also in the continuum system for the expectation values of functions $f\left(\left\{s_{c}(X ; \alpha, \omega)\right\}\right)$ and $g\left(\left\{s_{c}(X ; \alpha, \omega)\right\}\right)$ for which the corresponding lattice functions are monotone functions of $x$.

As an illustration of these inequalities we first note from (4.7) that $s_{\delta}(x ; 2, \omega) \geqq 0$ is an increasing function and $s_{\delta}(x ;-2, \omega) \geqq 0$ is a decreasing function of $x$. Hence, defining, in the usual way [15], the distribution function $n_{l_{1}, l_{2}}\left(\boldsymbol{x}_{1}, \ldots, \boldsymbol{x}_{l_{1}}, \boldsymbol{y}_{1}, \ldots, \boldsymbol{y}_{l_{2}} ;\left\{z_{\alpha}(\boldsymbol{r})\right\}, V\right)$ as the average joint density of having $l_{1}$ particles of species $B$ at positions $x_{1}, \ldots, x_{l_{1}}$ and $l_{2}$ particles of species $A$ at positions $\boldsymbol{y}_{1}, \ldots, \boldsymbol{y}_{l_{2}}, \boldsymbol{x}_{i} \in V$ and $\boldsymbol{y}_{j} \in V$, we have whenever $J(r) \geqq 4|\gamma(r)|$

$$
\begin{aligned}
& \left\langle\prod_{i=1}^{l_{1}} s_{c}\left(X ;-2, \omega_{i}\right) \prod_{j=1}^{l_{2}} s_{c}\left(X ; 2, \omega_{j}^{\prime}\right)\right\rangle \\
& \quad \equiv \int_{\omega_{2}} d \boldsymbol{x}_{1} \ldots \int_{\omega_{l_{1}}} d \boldsymbol{x}_{l_{1}} \int_{\omega_{1}^{\prime}} d \boldsymbol{y}_{i} \ldots \int_{\omega_{l_{2}}} d \boldsymbol{y}_{l_{2}} n_{l_{1} l_{2}}\left(\boldsymbol{x}_{1}, \ldots, \boldsymbol{y}_{l_{2}} ;\left\{z_{\alpha}(\boldsymbol{r})\right\}, V\right) \\
& \quad \leqq\left\langle\prod_{i=1}^{l_{1}} s_{c}\left(X ;-2, \omega_{i}\right)\right\rangle\left\langle\prod_{j=1}^{l_{2}} s_{c}\left(X ; 2, \omega_{j}^{\prime}\right)\right\rangle \\
& \equiv \int_{\omega_{1}} d \boldsymbol{x}_{1} \ldots \int_{\omega_{l_{1}}} d \boldsymbol{x}_{l_{1}} \int_{\omega_{1}^{\prime}} d \boldsymbol{y}_{1} \ldots \int_{\omega_{l_{2}}} d \boldsymbol{y}_{l_{2}} n_{l_{1}, 0}\left(\boldsymbol{x}_{1}, \ldots, \boldsymbol{x}_{l_{2}} ;\left\{z_{\alpha}(\boldsymbol{r})\right\} V\right) \\
& \quad \cdot n_{0, l_{2}}\left(\boldsymbol{y}_{1}, \ldots, \boldsymbol{y}_{l_{2}} ;\left\{z_{\alpha}(\boldsymbol{r})\right\}, V\right)
\end{aligned}
$$

where the sets $\left\{\omega_{i}\right\},\left\{\omega_{j}^{\prime}\right\}$ are assumed to be disjoint. Since (5.5) holds for all such domains $\left\{\omega_{i}\right\},\left\{\omega_{j}^{\prime}\right\}$ and the distribution functions exist and are piece-wise continuous (for a fixed volume $\mathrm{V}$ ) we must have

$$
n_{l_{1}, l_{2}}\left(\boldsymbol{x}_{1}, \ldots, \boldsymbol{x}_{l_{2}}, \boldsymbol{y}_{1}, \ldots, \boldsymbol{y}_{l_{2}}\right) \leqq n_{l_{1}, 0}\left(\boldsymbol{x}_{1}, \ldots, \boldsymbol{x}_{l_{1}}\right) n_{0, l_{2}}\left(\boldsymbol{y}_{1}, \ldots, \boldsymbol{y}_{l_{2}}\right) .
$$

The inequalities (5.6) apply in particular to the Widom-Rowlinson model and similar systems discussed by Lebowitz and Lieb [14] and by Stillinger and Helfand [5]. 
When there are hard core interactions between particles of the same species in the continuum system we are still able to obtain the modified inequalities of Section 4. We expect that the inequalities for continuum systems can be used, as they have been used for lattice systems, to establish the existence of the infinite volume limit of correlation functions in certain cases and to obtain inequalities between critical exponents. We plan to investigate this further.

Acknowledgments. We would like to thank R. Griffiths for a useful discussion of the FKG inequalities.

\section{References}

1. Griffiths. R.B.: Physica 33, 689 (1967) and references given there.

2. Sivardieve, J., Blume, M.: Phys. Rev. B, 5, 1126 (1972).

3. Bell, G. M., Lavis, D. A.: Phil. Mag. 11, 937 (1965) and references given there.

4. Wheeler, J.C., Widom, B.: J. Chem. Phys. 52, 5334 (1970), and references given there.

5. Helfand, E., Stillinger, F.H.: J. Chem. Phys. 49, 1232 (1968).

6. Blume, M., Emery, V. J., Griffiths, R. B.: Phys. Rev. A 4, 1071 (1971).

7. Griffiths, R. B.: J. Math. Phys. 10, 1559 (1969).

8. Griffiths, R.B.: J. Math. Phys. 8, 478, 484 (1967).

9. Kelly, D., Sherman, S.: J. Math. Phys. 10, 1559 (1969).

10. Fortuin, C.M., Kasteleyn, P.W., Ginibre,J.: Commun. math. Phys. 22, 89 (1971).

11. Lebowitz, J. L., Gallavotti, G.: J. Math. Phys. 12, 1129 (1971).

12. Widom, B., Rowlinson, J. S.: J. Chem. Phys. 52, 1670 (1970).

13. Ruelle, D.: Phys. Rev. Letters 27, 1040 (1971).

14. Lebowitz, J. L., Lieb, E.: Phys. Letters 39 A, 98 (1972).

15. Ruelle, D.: Statistical mechanics. New York: Benjamin 1969.

Joel L. Lebowitz

Belfer Graduate School of Science

Yeshiva University

New York, N.Y. 10033

USA 
